\title{
Uwb Bpf using Quad Section Stepped Impedance Resonator
}

\author{
Thomas George and Lethakumari B
}

\begin{abstract}
Ultra wide band (UWB) frequency range is from 3.1 to 10.6 $\mathrm{GHz}$ with a bandwidth of $7.5 \mathrm{GHz}$. UWB bandpass filter(BPF) is an essential component of any UWB communication system. Design of $U W B B P F$ with extremely high bandwidth is a challenge task. In this paper a simple method is developed for the design of UWB BPF. A quad section stepped impedance resonator (QSSIR) is used as the basic element of the filter. QSSIR has four impedance sections connected in cascade. Ultra wide bandwidth is realized by appropriately selecting impedance ratio and electrical length ratio of the SIR. Two transmission zeros are introduced in the insertion loss response by folding the QSSIR. Designed filter has bandwidth extending from 3.73 to $9.93 \mathrm{GHz}$, with a fractional band width (FBW) of $91 \%$ and center frequency $6.83 \mathrm{GHz}$. There are five transmission poles in the obtained frequency response. Simulation is performed using ANSOFT HFSS. Designed filter was fabricated using FR4 substrate. Test results are found to be in good agreement with simulations. Proposed filter has a simple planar structure. Fabrication is easy. It can be used in UWB systems as band pass filter.

Keywords: Fractional bandwidth (FBW), Multiple mode resonator (MMR), Quad-section SIR (QSSIR), Stepped impedance resonator (SIR), Uniform impedance resonator (UIR), Ultra wideband (UWB).
\end{abstract}

\section{INTRODUCTION}

Ultra wideband frequency band is from 3.1 To $10.6 \mathrm{GHz}$ [1]. U.S Federal Communication Commission (FCC) in 2002 legalized the use of UWB for license free applications. UWB provides high data rate in the range $110 \mathrm{Mb} / \mathrm{s}$ with very low power. Some of the applications of UWB band are position location, tracking, through wall imaging, medical imaging, wireless sensors, low power RFID tags and high speed wireless USB. Important advantages of this system are wide bandwidth, low power requirement and large throughput. According to FCC, the indoor and hand held operations of UWB must be strictly restricted in the range of 3.1 to 10.6 $\mathrm{GHz}$. For implementing this frequency limit, bandpass filter is an essential building block in all UWB systems. Such an

Revised Manuscript Received on November 19, 2019

* Corresponding Author

Thomas George*, Research Scholar, Electronics and Communication Engineering Department, University College of Engineering, Thodupuzha, Kerala, India

Dr.Lethakumari. B, Associate Professor, Electronics and Communication Engineering Department, University College of Engineering, Thodupuzha, Kerala, India.
UWB BPF must have a fractional bandwidth of $109.5 \%$ with a center frequency of $6.85 \mathrm{GHz}$. A large number of diverse methods are available for UWB bandpass filter design in the literature. A filter using a multi mode resonator which is a stepped impedance resonator, coupled to the input output through parallel lines is capable of producing UWB response in the range 3.1-10.6 GHz [2]. A very simple method of UWB filter design is based on parallel coupled lines [3]. Coupling between lines is to be made tight for wideband response [4]. Tight coupling can be obtained by reducing the spacing between lines. Minimum realizable coupled space is dependent on the fabrication process. Sophisticated techniques are required for very narrow line spacing. High pass and low pass filters using liquid polymer technology are cascaded for achieving UWB response in [5]. A hybrid UWB filter is proposed in [6] using a CPW non-uniform resonator. In [7] a compact UWB BPF design is reported by connecting a short ended coupled line coupler and a transmission line in parallel.This paper proposes a very simple design approach for UWB BPF design. Proposed filter uses a single quad section SIR. QSSIR is folded and is connected to $50 \Omega$ input output feed lines. SIR operating as multiple mode resonator (MMR) helps to keep the structure compact. SIR folding leads to further reduction of the filter size. Folding also results in two signal travelling paths from input to output. This multipath introduces two transmission zeros, one each at the two edges of UWB passband. In order to obtain a wideband response, a number of higher order resonances of the SIR are located within the passband. It is to be noted that, the designed filter has a very simple structure.

\section{STEPPED IMPEDANCE RESONATOR}

Makimoto and Yamashita in 1980 [8] first proposed SIR which is a logical extension of uniform impedance resonator (UIR). It is a multi mode resonator consisting of two composite transmission lines in cascade [9]. Simple two section SIR has two impedance sections $Z_{1}$ and $Z_{2}$ with electrical lengths $\theta_{1}, \theta_{2}$ connected together. Main advantages of SIR are compact size and independently tunable resonant frequencies. In addition to the above mentioned features, BPFs based on SIRs usually have high selectivity and low insertion loss. Hence SIRs are widely used for BPF design. Structure of a simple SIR is shown in Fig.1. 


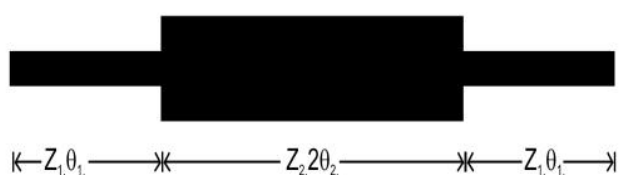

Fig.1. Simple SIR

The two important parameters which characterizes the SIR are the impedance ratio $\mathrm{k}$ defined as $\mathrm{k}=\frac{z_{1}}{z_{z}}$ and the electrical length ratio $\alpha=\frac{\theta_{2}}{\theta_{1}+\theta_{2}}$. The input admittance of SIR is given by,

$$
Y_{\text {in }}=j \frac{Z_{2} \tan \theta_{1} \tan \theta_{2}-Z_{1}}{Z_{1}\left(Z_{1} \tan \theta_{1}+Z_{2} \tan \theta_{2}\right)}
$$

From (1) it is clear that the resonant behavior of the SIR can be greatly controlled by varying the parameters $\mathrm{k}$ and $\alpha$ [8]. Fig.2 shows the structure of a quad section SIR (QSSIR) which is constituted by four impedance sections of $Z_{1}, Z_{2}, Z_{3}$ and $Z_{4}$ with electrical lengths $\theta_{1}, \theta_{2}, \theta_{3}$ and $\theta_{4}$. Asymmetric form of the above QSSIR is shown in Fig.3 and when folded it will appear as in Fig.4.

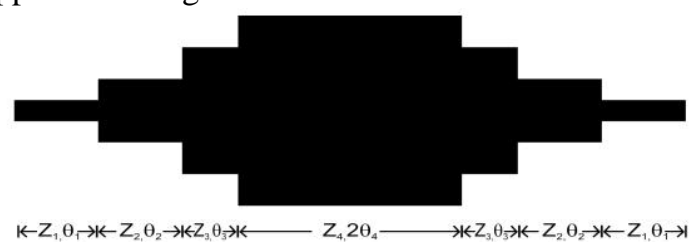

Fig.2. QSSIR

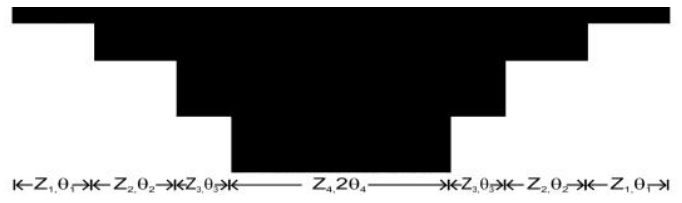

Fig 3. Asymmetric QSSIR

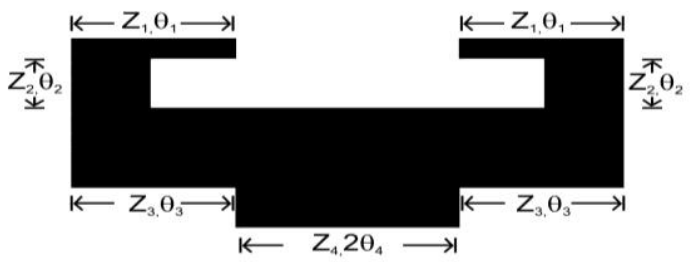

Fig 4. Folded QSSIR

\section{PRoposed UWB BPF}

Structure of the proposed UWB BPF filter, which consists of a single QSSIR is given in Fig.5. Initially the SIR is appropriately tuned to get a flat S21 response. Then the tuned SIR is folded and two transmission zeros appear at both sides of the frequency response. This limits the passband into ultra wide band, $3.1 \mathrm{GHz}$ to $10.6 \mathrm{GHz}(7.5 \mathrm{GHz})$. Transmission zeros are formed due to signal multipath from input to output. Optimization of the designed structure is done using HFSS. Input output feed lines with $50 \Omega$ impedance are connected to the folded SIR.

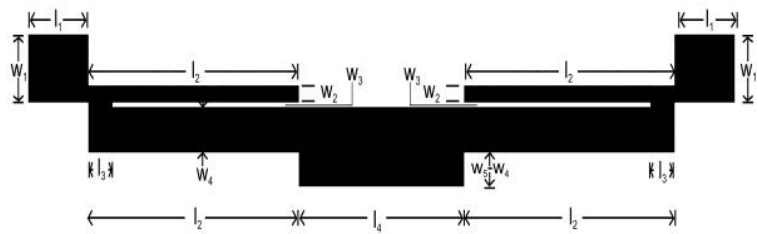

Fig.5. UWB BPF using QSSIR
The QSSIR used is having four impedance levels. Different parameters of the QSSIR are $\mathrm{Z1}=97.73 \Omega, \mathrm{Z} 2=70.83 \Omega, \mathrm{Z} 3=$ $63.57 \Omega, Z 4=46.27 \Omega, \theta 1=171.06^{\circ}, \theta 2=2.50^{\circ}, \theta 3=176.42^{\circ}, \theta 4=$ $70.86^{\circ}, \mathrm{k} 1=1.38, \mathrm{k} 2=1.11$ and $\mathrm{k} 3=1.37$. Dimensions of the filter are, $11=4 \mathrm{~mm}, 12=14 \mathrm{~mm}, 13=1.6 \mathrm{~mm}, 14=11 \mathrm{~mm}, \mathrm{w} 1=3 \mathrm{~mm}$, w2 $=0.75 \mathrm{~mm}, \mathrm{w} 3=0.2 \mathrm{~mm}, \mathrm{w} 4=2 \mathrm{~mm}, \mathrm{w} 5=3.5 \mathrm{~mm}$. Passband of the filter is derived from five resonant frequencies from 3 rd onwards. Resonant frequency locations are listed in Table 1.

Table 1. Resonant frequency locations

\begin{tabular}{|c|c|}
\hline \multicolumn{2}{|c|}{ Transmission } \\
Pole locations in GHz \\
\hline Below the & 1.55 \\
passband & 2.6 \\
\hline In the pass & 4 \\
Band & 4.89 \\
& 6.76 \\
& 8.57 \\
& 9.75 \\
\hline Above the & 10.23 \\
Passband & 10.63 \\
\hline
\end{tabular}

\section{RESULTS AND DISCUSSIONS}

The proposed filter was simulated using Ansoft HFSS 3D full-wave electromagnetic field simulation software version 13.0 based on finite element method (FEM). Filter was fabricated using conventional FR4 PCB board with dielectric constant $\varepsilon_{\mathrm{r}}=4.4$, dielectric loss tangent $\tan \delta=0.02$ and with a thickness of $\mathrm{h}=1.6 \mathrm{~mm}$. Input and output ports are connected to SMA connectors for measurement. Photograph of the fabricated filter with SMA connectors is shown in Fig.6. Ground plane area of the filter is only $47 \mathrm{~mm}$ x $11 \mathrm{~mm}$ $=517 \mathrm{~mm}^{2}$. Fabricated filter was tested using KESIGHT E5063A (100 KHz - $18 \mathrm{GHz})$ Series Network Analyzer.

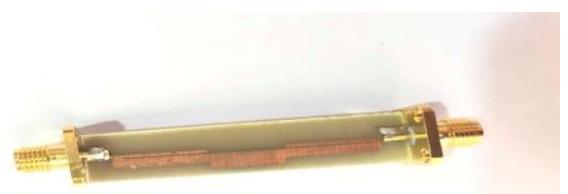

Fig.6. Photographs of fabricated filter

Responses of the simulated and fabricated filters are shown in Fig.7. Simulated band pass response is from 3.73 to $9.93 \mathrm{GHz}$ $(6.20 \mathrm{GHz})$ and measured 4 to $9 \mathrm{GHz}(5 \mathrm{GHz})$. Comparison of various parameters of simulated and fabricated filters are given in Table 2. Some of the reasons for deviations in the measured results are tolerances in the fabrication process, SMA connectors, radiation loss and soldering.

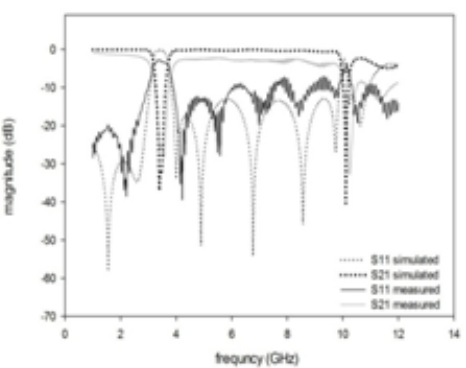

Fig.7. Filter response 
Insertion loss is found to be increasing with frequency increase. It is mainly attributed to variation of material characteristics with frequency.

Table 2. Comparison of parameters of the filter

\begin{tabular}{|c|c|c|}
\hline Parameter & Simulated & Measured \\
\hline $\begin{array}{c}\text { Lower cut off } \\
\text { frequency }\end{array}$ & $3.73 \mathrm{GHz}$ & $4.00 \mathrm{GHz}$ \\
\hline $\begin{array}{c}\text { Upper cut off } \\
\text { Frequency }\end{array}$ & $9.93 \mathrm{GHz}$ & $9.00 \mathrm{GHz}$ \\
\hline Center frequency & $6.83 \mathrm{GHz}$ & $6.50 \mathrm{GHz}$ \\
\hline Bandwidth & $6.20 \mathrm{GHz}$ & $5.00 \mathrm{GHz}$ \\
\hline $\begin{array}{c}\text { Location of first } \\
\text { transmission zero } \\
\text { and insertion loss }\end{array}$ & $\begin{array}{c}3.40 \mathrm{GHz} \\
37 \mathrm{~dB}\end{array}$ & $\begin{array}{c}3.56 \mathrm{GHz} \\
28 \mathrm{~dB}\end{array}$ \\
\hline $\begin{array}{c}\text { Location of second } \\
\text { transmission zero } \\
\text { and insertion loss }\end{array}$ & $10.11 \mathrm{GHz}$ & $10.25 \mathrm{GHz}$ \\
\hline $\begin{array}{c}\text { Maximum in band } \\
\text { insertion loss }\end{array}$ & $0.64 \mathrm{~dB}$ & $3.93 \mathrm{~dB}$ \\
\hline $\begin{array}{c}\text { Minimum in band } \\
\text { return Loss }\end{array}$ & $-13 \mathrm{~dB}$ & $-7.90 \mathrm{~dB}$ \\
\hline $\begin{array}{c}\text { Fractional band } \\
\text { width }\end{array}$ & $91 \%$ & $77 \%$ \\
\hline
\end{tabular}

\section{CONCLUSION}

A simple UWB BPF is designed using a single folded quad section SIR. Ultra wide band response is achieved by tuning the two SIR parameters, impedance ratio and electrical length ratio. Folding of the QSSIR leads to multipath and two transmission zeros are generated, which limits the response into ultra wide bandwidth. Experimental verification is done by fabricating and testing the filter. Test results are found to be in good agreement with simulations. Designed filter has the advantages of simple structure, low cost, small size, good skirt selectivity, low insertion loss and wide passband. Since no via holes and defected ground structures are used, fabrication of the filter is easy. This filter can be used as bandpass filter in UWB communication systems.

\section{REFERENCES}

1. United States Federal Communication Commission (FCC, www.fcc.gov), Revision of Part 15, the Commission's Rules Regarding to Ultra Wide Band Transmission System, First Note and Order Federal Communication Commission, ET-Docket 98-153, 2002.

2. L. Zhu, S. Sun and W. Menzel, "Ultra-Wideband (UWB) Bandpass Filters using Multiple-Mode Resonator", IEEE Microwave \& Wireless Component Letters, Vol. 15, No. 11, 2005, pp. 796-798.

3. R. Xiao, G. Yang , Y. Wang and W. Wu, "Compact and High Selectivity Dual Band Dual Mode Microstrip BPF with 5 Transmission Zeroes", IEEE International Symposium on Microwave, Antenna, Propagation and EMC Technologies, Oct 2017, pp.300-302

4. D. -M. Pozar, "Microwave Engineering", $2^{\text {nd }}$ ed., New York: Wiley 1998

5. Z. Hao and J. Hong, "UWB Bandpass Filter using Cascaded Miniature High Pass Low Pass Filters with Multilayer Liquid Crystal Polymer Technology", IEEE Transactions on Microwave Theory and Techniques, Vol.58, no.4, April 2010, pp.941-948

6. H. Wang, L. Zhu and W. Menzel, "Ultra-wideband bandpass filter with hybrid microstrip/CPW structure", IEEE Microwave and Wireless Component Letters, Vol. 15, No. 12, 2005, pp. 844-846.

7. A. Miguel, E. Bronchalo and G. T. Penalva, "Compact UWB Bandpass Filter Based on Signal Interference Technique", IEEE Microwave and Wireless Components Letters, Vol.19, No.11, Nov.2009,pp.692-694.
8. M. Makimoto and S. Yamashita, "Microwave Resonators and Filters for Wireless Communication Theory, Design and Application", Springer, Japan,2000, Ch.2.

9. F. Sakai, M. Makimoto and K. Wada, "Multimode Stepped Impedance Resonators and Their Applications in Chipless RFID Tags", European Microwave conference, 2016.

\section{AUTHORS PROFILE}

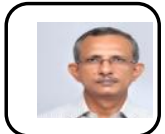

Thomas George is working as Associate Professor in the Department of Electronics and Communication Engineering, Mar Athanasius College of Engineering, Kothamangalam, Kerala, India. He completed his B.Tech in Electronics and Communication Engineering from Kerala University in 1990 and M.Tech in Communication Systems and High Frequency Technology from I.I.T., Madras in 1996. He is presently persuing his Ph.D in M.G.University, Kerala, India under the guidance of Dr.Lethakumari B, Professor and HOD, Electronics and Communication Engineering, Univesity College of Engineering, Thodupuzha, Kerala, India He is a life member of Indian Society for Technical Education and a Fellow of Institution of Engineers, India.

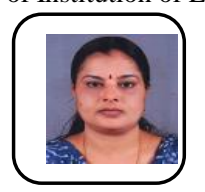

Dr.Lethakumari. B is working as Associate Professor Electronics and Communication Engineering Department of University College of Engineering, Thodupuzha, Kerala, India. She has a Ph.D in Microwave Electronics from The Department of Electronics, Cochin Universiy of Science and Technology, Kochi, Kerala. She completed her B.Tech in Electronics and Communication Engineering from Kerala University in 1991 and M.Tech in Microwave and TV Engineering from Kerala University in 1994. She has publications in 6 international journals and 4 national journals. She is a member of IEEE and Indian Socitey for Technical Education. Dr.Lethakumari is a research guide in Mahatma Gandhi University, Kerala. 